\title{
La lengua en disputa: los debates en la prensa escrita y una historia de la lengua para el siglo XXI
}

Language in dispute: Debates in the written press and a language history for the 21 st century

\author{
Juan Antonio Ennis \\ CONICET / IdIHCS, Facultad de Humanidades y Ciencias de la Educación \\ Universidad Nacional de La Plata, Argentina
}

Guillermo Toscano y García

Instituto de Lingüística - Universidad de Buenos

Aires, Argentina

El punto de partida es relativamente sencillo y verificable desde el sentido común más lego: el lenguaje todo lenguaje, con Lotman (1988), todo sistema de modalización- cambia cuando cambian las condiciones técnicas y políticas de su existencia. Recordando la vieja, tan amplia como precisa definición de Raymond Williams (1977), si hablar del lenguaje supone hablar de los seres humanos en el mundo, cuando el modo de estar en el mundo cambia radicalmente también lo hace el modo de vivir en y a través del lenguaje. Con esto queremos decir que lo que cambia no es solamente la forma fonética, morfosintáctica o pragmáticosemántica más o menos aislable de una lengua dada, sino más aún el modo en el cual el lenguaje se hace vivible, articulándose política y socialmente.

En este sentido, la historia moderna es también la historia de las lenguas modernas como atributo esencial del sujeto de la historia que se identificaba en la nación, y el dispositivo que las hace posibles, la imprenta, y sobre todo sus avatares modernos como la novela o el periódico, aquello que hizo posible pensarlas como formas "estandarizadas", uniformes o capaces de ser masiva, taylorísticamente uniformadas ${ }^{1}$, estandarizadas, ofreciendo un plano tan simultáneo como paradójico de igualdad y sujeción posible.

Hoy que los debates del día nos devuelven a la conciencia de lo históricamente situado y contingente de los regímenes mismos de normatividad (v. Arnoux y del Valle 2010) en disputas sobre los modos de visibilizar la inscripción del poder en el lenguaje y al mismo tiempo sublevarla --aquello que se ha dado en llamar la polémica por el lenguaje inclusivo- y que la vertiginosa carrera de una tecnología que cambia constantemente el soporte de la comunicación y con él sus formas, límites y modos de darse o prescindir de normas, las mismas circunstancias políticas y el mismo cambio tecnológico nos obligan y permiten, de un modo radicalmente amplio y novedoso, interrogar las formas históricas de lo que conocimos como lengua en su materialidad.

Así, en las últimas décadas asistimos a una verdadera fiebre de archivo en las humanidades en general y en los estudios históricos de la lengua y sus saberes en particular. Diluyendo poco a poco los porosos límites de la lingüística externa e interna, que durante más de un siglo habían trazado la frontera entre la legitimidad científica y el diletantismo, distintas disciplinas comienzan a colaborar en la construcción de un corpus que no pretende aislar la materia significante de su contexto, sino que toma en cuenta precisamente las circunstancias de su ocurrencia, las diversas formas de su soporte material e institucional, para dar forma a lo que con fortuna se nombró, en un reciente volumen colectivo, la historia política de la lengua (Del Valle ed., 2013, 2015). La historia política del español como lengua moderna mayoritaria resulta de este modo indesligable (al igual que la de la mayor parte de las lenguas llamadas modernas) de la historia de los Estados-nación en los que se habla, escoge y construye como lengua de la prensa, la literatura, la administración y la educación. Así, difícilmente pueda completarse un panorama de la historia de la lengua en España y América Latina sin tener en cuenta la serie de procesos históricos que instalan el horizonte de la nación monolingüe como proyecto de Estado 
y el modo en el cual el discurso y las políticas sobre la lengua se ubican en el centro de las estrategias de las élites dominantes para la construcción de hegemonía, es decir, entre otras cosas, para hacer de ese horizonte un artículo del sentido de lo común, y cómo en esa discusión se construyen puentes y alianzas, se desatan conflictos y se declaran rupturas entre España y América.

Si, de acuerdo con Paul V. Kroskrity (2006: 501), las ideologías lingüísticas representan, en una primera acepción, "the perception of language and discourse that is constructed in the interest of a specifical social or cultural group", el de la prensa escrita se ofrece como un espacio insoslayable para investigar el modo en el cual esas percepciones entran en tensión o en pugna según el caso. De acuerdo con el clásico estudio de B. Anderson (1993 [1983]), la prensa y la novela son fundamentales para el desarrollo del print capitalism que hace posible la forma moderna de la nación; en ese entramado será de fundamental importancia la lengua nacional, su concepción y alcances, que es parte de un paquete que alcanza en distintas versiones y apropiaciones los más recónditos rincones del globo, y especialmente aquellos más sensibles. Por otra parte, como lo han establecido trabajos clásicos como los de Lucien Febvre y Henri-Jean Martin (1958), Elisabeth Eisenstein (2005: 5, 6, 53-56), Silvain Auroux (1994) o Peter Burke (2004: 91 sigs.), el cambio tecnológico que supone la irrupción, modernización y expansión de la prensa escrita resulta determinante en la producción de la forma moderna de las lenguas nacionales y su horizonte de estándar, que solo se hace realmente pensable a partir de la producción masiva y en serie de textos de disposición idéntica hasta en sus yerros.

Así, es en el terreno de la prensa donde se encuentra una de las superficies fundamentales para la construcción de lo nacional y sus deslindes, así como de la maquinaria de lo público para la afirmación del Estado, lo cual implica que sea en ese entramado que se propicie la mediatización y circulación de ideologías lingüísticas. En consecuencia, podemos anticipar que serán los debates en torno a la lengua nacional (no solo alrededor de su condición, nombre, características y necesidad, sino también en torno a su estado, decadencia, progreso o promisorio futuro) los que predominen en ese terreno y concentren, aunque no exclusivamente, el lugar ocupado por las ideologías lingǘsticas en la prensa escrita, siendo que todo a lo largo del período consignado intervenciones asociadas a constructos ideológicos en principio no exclusivos de este ámbito (como el purismo) derivan forzosamente en la discusión por la lengua nacional y sus saberes más o menos especializados según el caso. De tal forma, el volumen monográfico que proponemos busca concentrar su atención en esta problemática, la de los debates por la unidad de la lengua, que atraviesan todo el proceso de modernización a lo largo del siglo XIX y XX entre España y América; con ello, pretende aportar al conocimiento creciente que sobre el tema ha venido generándose durante los últimos años, atendiendo especialmente al modo en que estos debates se articulan en el marco de la prensa escrita en el mundo de habla hispana.

El monográfico se integra en el desarrollo del PICT 2014-1688, "Ideologías lingüísticas en la prensa escrita en Argentina (1810-1930): Corpus, teorías, métodos”, proponiendo así la presentación de los distintos avances logrados en el marco de dicha investigación, puestos en diálogo en un marco más amplio en su alcance histórico y geográfico con el trabajo desarrollado por especialistas internacionalmente reconocidos en las distintas ramas de la investigación que convergen en este tipo de problemáticas: la historiografía lingüística, la glotopolítica, los estudios de ideologías lingüísticas, los de la prensa y la literatura, los de las instituciones de la cultura y la historia cultural e intelectual, entre otras.

El campo de estudio dedicado a las ideologías lingüísticas encuentra un terreno de especial riqueza para su trabajo en el espacio de la prensa escrita, que es justamente uno de los ámbitos fundamentales, junto al de la educación, en que se definen la forma y los límites del mercado de los bienes simbólicos (en términos de Bourdieu), en el cual la forma de la lengua legítima constituye un valor central, verdadero índice de pertenencia en algunos casos. En este sentido, si el desarrollo de la prensa periódica -tal como se viene discutiendo desde ya clásicos e imprescindibles trabajos como los de Jürgen Habermas([1968] 1981) o Benedict Anderson([1983] 1993) - resulta fundamental para la construcción de las naciones y nacionalismos 
modernos, será en las páginas periódicas de esa prensa, de la prensa cultural y de la prensa especializada donde el debate por la forma de la lengua legítima para la nación encuentre un primario y, políticamente hablando, decisivo alcance y desarrollo. Este debate se hace particularmente intenso en el proceso de modernización y consolidación del Estado en Argentina, fundamentalmente entre 1880 y 1945, y concierne tanto a la típica discusión de las formas de la lengua legítima (la "complaint tradition" de Milroy y Milroy 1985, en la que la prensa periódica y la participación de los lectores juegan un rol importante) como a la discusión por la autoridad en materia lingüística, la legitimidad de las diferentes variedades y formas del contacto (sobre el valor de la lengua propia en su diversidad y sus "otros": lenguas indígenas, migratorias, variedades de contacto) $y$, sobre todo, la autoridad sobre la lengua y el futuro del español. En el momento en que parece ser la forma de la nación y los límites del Estado lo que entra en disputa, la discusión de laS lenguaS se vuelve central. Como se enuncia más abajo, para que tales desarrollos se hagan posibles, resulta necesario un proceso de estandarización en sentido amplio (que incluye la estandarización de las variables lingüísticas) cuyo soporte fundamental se encuentra en las prácticas públicas y privadas vinculadas a la llamada "cultura impresa" (v. Acree 2013), que articula la tecnología de la imprenta y la materialidad del impreso con la incidencia de la creciente expansión y presencia del espacio y las prácticas asociadas a la institución escolar. En efecto, tal como han demostrado muchos estudios en relación con la formación de la literatura moderna (vgr. Viala 1985, Bourdieu 1992, Adamowicz-Hariasz 1999), la prensa periódica hizo posible la difusión y asimilación por parte de un público lector cada vez más amplio de una forma lingüística estandarizada, a la vista y al oído.

Si se pretende dar cuenta, entonces, del desarrollo de una lengua estándar pluricéntrica como la española en la historia ${ }^{2}$, debe tenerse en cuenta no solo el modo en el cual los distintos centros consolidados como emisores de la norma y proveedores de prestigio lingüístico entran abierta o solapadamente en tensión y eventual disputa (lo que viene siendo estudiado en los debates ideológico-lingüísticos que atraviesan el espacio de la prensa escrita en el período), sino también el modo en el cual emergen las diversas estructuras centradas y en ellas los centros de configuración, afirmación o difusión de la norma. Para ello, las condiciones sociales, políticas, económicas y tecnológicas de la afirmación de un centro urbano como espacio de discusión y emisión de normas no solamente lingüísticas, propicio sobre todo para la producción del orden material que favorece la afirmación del estándar como variante "normal" de la praxis lingüística, resultan una variable de relevancia para el examen lingüístico. En el corpus de debates sobre la lengua y sus saberes, en lo que la glotopolítica llama las “intervenciones sobre el espacio público del lenguaje”, en este caso precisamente desde la prensa, esta preocupación por la normatividad, por las decisiones en torno a la gestión de la lengua como forma pública (la expresión es de Quintiliano, v. Ennis 2014) resultará una constante.

De este modo, hablar de una "historia política de la lengua" implica hoy en día una toma de posición no solo en cuanto a decisiones epistemológicas precisas en el ámbito de los estudios del lenguaje, sino también en cuanto a los límites mismos fijados para investigaciones planteadas inicialmente al interior del mismo, pero que necesariamente deben excederlo, explotando la porosidad de sus límites. La primeramente $A$ Political History of Spanish (2013), luego traducida como Historia politica del español (2015) dirigida por José del Valle vino a corroborar algo que habían sabido anticipar las distintas vertientes más o menos heterodoxas de los estudios lingüísticos (antropología lingüística, sociolingüística histórica, análisis crítico del discurso, glotopolítica, entre otros), dándole un espacio de especial visibilidad en el horizonte de la historia de la lengua: que cualquier mirada sobre la historia de la lengua, si quería ser histórica, debía comenzar por superar, aboliéndola, la escisión que la había fundado, aquella que consagraba la separación discreta entre historia lingüística interna e historia lingüística externa (v. Blumenthal 2003) que ya Coseriu supo censurar en sus bases mismas (1978: 16). Así, pensar la historia de la lengua en términos de una historia política supondría, con Crowley (1996), una manera clara y decidida de pensar la lengua en la historia. La premisa en este caso, en síntesis, establece que el sustrato empírico sobre el cual se ejerce la indagación histórica del objeto lengua (de una lengua en particular, de varias lenguas comparadas, con Foucault, del modo mismo de ser del lenguaje) comprende tanto el aislamiento de las formas gramaticalmente relevantes como los discursos sobre 
la lengua, aquellos que permiten la delimitación de esas formas, su disposición en un mapa necesariamente (gloto)político ${ }^{3}$.

De este modo, al proponer un aporte a la historia política de "la" lengua, nos referimos a la historia de la lengua como línea fundamental de la historia política. En este sentido, cabe recordar que el desarrollo de la cultura monoglósica como módulo central del Estado-nación, consecuencia de la transferencia de soberanía operada por la Revolución Francesa, apela a la figura de pueblo soberano comprendido en tanto público, es decir sujeto de un novedoso espacio público conformado por la audiencia ${ }^{4}$ de un espacio común cuyo sostén material principal y a la vez secularizante lo provee la cultura impresa, y cuya comunidad demanda, en consecuencia, la estandarización de sus códigos (legales, lingüísticos, entre otros). El vínculo entre lengua y nación viene dado por la posibilidad de producir un estándar. En este sentido, el fenómeno que conocemos bajo el nombre de "estandarización" no es privativo de los estudios lingüísticos sino que tiene una larga tradición en los trabajos de la nueva historia cultural, en particular la referida al desarrollo de la imprenta y sus consecuencias. En su discusión del concepto de standard, James Milroy (2001: 531) provee una definición inicial que resulta bien ilustrativa en este caso -para comenzar a discutir un concepto que, como ha demostrado recientemente Smakman (2012), no parece ser claro ni unívoco en su definición tanto para legos como para especialistas-: la estandarización consistiría así en primera instancia en "la imposición de uniformidad sobre una clase de objetos". Ese sencillo principio es el que vemos operar en lo que se conoce como la ideología lingüística del homogeneism (Blommaert y Verschueren 1998: 202-204), que Blommaert sintetiza como "una ideología en la cual la homogeneidad social, cultural, lingüística y de otro tipo se presenta como la 'mejor' forma de gubernamentalidad [governance]” (2005: 252) , y cuya definición y descripción en el ámbito de la lengua española se hacen más precisas luego con la introducción del concepto de "cultura monoglósica" (ver, entre otros, Del Valle y Stheeman 2002: 10-11).

El espacio privilegiado para el desarrollo de este proceso es el de la escuela, y su tecnología la imprenta. Como puede leerse en el clásico estudio de B. Anderson (1983), la prensa y la novela aparecen como superficies fundamentales para el desarrollo del print capitalism, en cuya lógica la lengua, que será a su vez la de la educación y la administración, juega un rol determinante. La formación de lectores y el desarrollo técnico que hace posible la producción masiva del impreso contribuyen a la formación de un mercado de la lectura que, como todo mercado, requiere de una superficie homogénea, una moneda de cambio, de modo tal que ambos, educación formal generalizada y mercado de la letra impresa, se condicionan mutuamente ${ }^{6}$. Es por ello que los debates sobre la lengua en la prensa conviven y se confunden al mismo tiempo tanto con aquellos sobre la posibilidad, necesidad y alcance de una literatura nacional, como con aquellos en torno a la introducción del saber especializado sobre su lengua. Como observa Susan Gal (1989: 355) comentando el clásico estudio de Anderson, el vínculo entre lengua y nación no estaría dado tanto por la preexistencia de una lengua compartida como por "los efectos unificadores de la imprenta". Así, por un lado, la posibilidad y necesidad de imprimir una cantidad cada vez mayor de libros idénticos para dar forma a un mercado del impreso afirmó las murallas entre naciones y lenguas (y entre las naciones a través de las lenguas); mientras, por el otro, la prensa escrita y los instrumentos lingüísticos de difusión masiva ${ }^{7}$ cumplirían con eficiencia la tarea de la imposición de esa uniformidad.

El proyecto de este monográfico -que por las características de las investigaciones de los autores individuales que reúne ofrece una particular concentración de la atención en el espacio argentino- no puede sino pensarse entre espacios y entre tiempos, en el tránsito entre formas de organización política y social que se da entre España y América a lo largo de los siglos XIX y XX. La lengua en disputa es la castellana, cuyas exequias ponía en escena ya a fines del XVIII Juan Pablo Forner, y que la generación argentina del 1837, haciéndose eco de su admirado Larra, declaraba inútil para entrar en la modernidad; pero también lo son las lenguas indígenas habladas en el país. Sin embargo, el análisis que ofrecen los artículos contenidos en este dossier no se limita a la exposición de argumentos históricos acerca de las ventajas o desventajas de la 
unidad o disgregación lingüística del antiguo imperio, sino que se detiene con mayor precisión en las formas de intervenir en el modo en el cual esa, la lengua del estado y la nación, se desplegará en un entramado social cambiante, convivirá con otras lenguas, distribuirá autoridades.

En primer lugar, el trabajo de Elvira Narvaja de Arnoux, "La prensa escrita en manuales de retórica del siglo XIX: deslindes genéricos e ideologías lingüísticas”, se ocupa de examinar el modo en el cual un género particularmente influyente en el siglo XIX como el de las retóricas va incorporando en distintos momentos, progresivamente, los géneros periodísticos. Inicialmente pensadas tanto para la educación formal como para la elaboración de discursos públicos orales y escritos, el desarrollo y expansión de la prensa escrita hace que estos manuales comiencen a hacer lugar al tratamiento de los géneros periodísticos. En un comienzo, estos no llegan a ser apreciados en su especificidad y se incorporan integrándose a otros registros -como la elocuencia política, la prosa didáctica o los textos históricos- y esta perspectiva se continúa en numerosos tratados posteriores producidos, incluso, a comienzos del siglo XX. No obstante, existen excepciones notables, como la de Vicente Fidel López, quien a mediados del XIX llama ya la atención sobre la necesidad de tomar en consideración en la retórica los distintos tipos de publicaciones y los rasgos de los géneros que transitan por sus páginas. Así, Arnoux prosigue el examen de distintos tratados que, sensibles a la importancia adquirida por la prensa industrial y el desarrollo del periodismo de información, comienzan posteriormente a contemplar una mayor variedad de tipos de textos, de acuerdo a la mayor variedad de secciones que comienza a incluir la prensa, proponiendo caracterizaciones rápidas de algunas de ellas. El artículo ofrece un análisis de estas distintas etapas, poniendo el foco sobre las ideologías lingüísticas que movilizan, tanto en lo relativo a la función social de la prensa como también a los géneros que se incluyen en los tratados, y observando finalmente cómo el dispositivo normativo retórico, si bien sirvió para pensar la escritura periodística, no deja de constituir un obstáculo, en tanto y en cuanto no considera la práctica efectiva de las redacciones y la importancia que adquiere la figura del lector. A estos aspectos atenderán, en cambio, los manuales de periodismo que se publican desde la última década del siglo XIX.

En "El orden bellista. Purismo idiomático e intervención gráfica en la prensa temprana de Buenos Aires (1801-1830)”, Hernán Pas parte también de la articulación conflictiva entre el orden de la retórica y las nuevas prácticas y formas que impone el desarrollo de la prensa escrita en el siglo XIX, sobre todo en Chile y Argentina. Considerando a la prensa como plataforma central de la construcción pública de la lengua legítima en ese entonces, el trabajo de Pas revisa, por un lado, las intervenciones puristas y supervisoras del idioma previas a la intervención señera de Andrés Bello (tanto en Londres como en Chile) sobre el tema, observando sobre todo las intervenciones en periódicos de las primeras décadas postemancipatorias en el Río de la Plata, como El Censor, El Argos de Buenos Aires o El Tiempo. Por el otro, indaga, en un corpus más amplio de periódicos sudamericanos y en un período más extendido, el modo en el cual se produce el impacto material de la imprenta en la construcción de sentidos alrededor de la lengua. En este sentido, cobra especial importancia la preocupación en la prensa sudamericana por la construcción visual de sentidos, que Pas explica como correlato de la cultura libresca y del proceso de textualización de la lengua que la acompaña, y a la cual la prensa otorgará un alcance (con todas las limitaciones históricas del caso) por primera vez masivo.

María López García, por su parte, nos presenta un corpus de trabajo más tardío, ya entrando al siglo XX, y un género de prensa escrita novedoso para la época y como objeto de análisis en este contexto. Se trata de un estudio de la configuración de un público lector infantil en Argentina a partir del examen del caso de la pionera revista Pulgarcito (1904-1907). Sobre la base de un corpus de 16 números y en el marco que ofrece la glotopolítica, López García procede a interrogar el archivo de la prensa infantil a partir de sus distintos componentes: cómo se pensaba una revista, cuáles son sus condiciones de posibilidad, en qué momento y a partir de cuáles circunstancias puede pensarse en un público lector infantil, cómo se piensa a ese niño y cómo se lo interpela. La relación entre infancia, familia, Estado en la educación y los discursos circulantes en la esfera pública resulta así de importancia medular para pensar las características de la revistas y las formas de lectura y subjetividades lectoras que viene a proponer. El trabajo se propone así demostrar que la explotación 
del segmento infantil comenzaría entonces una carrera, actualmente en vigor, en la formación del niño como consumidor, y que la prensa infantil argentina de fines del XIX y comienzos del XX jugaría un rol central en la difusión de ese modelo de infancia. Finalmente, analiza la gestión de contenidos vinculados con la modelación del ciudadano, la propuesta de nuevas formas de lectura extraescolares, y la presencia de distintas pautas lingüísticas como ensayos de lo que pronto se constituiría como forma lingüística de la prensa infantil en Argentina. En este sentido, al concluir situando a la revista "en el umbral que divide al juego del mundo escolar" puede dar cuenta de cómo en lo tocante a la lengua Pulgarcito se mantiene en una indefinición u oscilación entre las variantes de la oralidad urbana y las sancionadas por la escuela que pone de manifiesto "un problema que la prensa debía entonces resolver: la necesidad de definir cuáles serían las formas lingüísticas del ocio infantil".

El trabajo de Luisa Domínguez es el único entre los que integran el presente monográfico que se centra en el estudio de las lenguas indígenas habladas en la Argentina. En particular, la autora aborda el debate que, durante la primera mitad del siglo pasado, se produce entre americanistas y lingüistas en relación con el estudio de estas lenguas. Ese debate, que Domínguez reconstruye a partir del riguroso examen de un conjunto de intervenciones que una serie de especialistas (Arturo Costa Álvarez, José Imbelloni, Enrique Palavecino y Carlos Vega) despliega en el ámbito de la prensa periódica (el diario La Prensa y la revista Nosotros, centralmente), le permite demostrar no solo que, aunque mayoritarios, los debates sobre el español no son los únicos que se producen durante el período, sino también y fundamentalmente el modo en que las disciplinas antropológicas y lingüísticas se relacionan, delimitan y reconfiguran durante el período. Ese proceso de redefinición implica, como lo demuestra Domínguez, una discusión de las teorías lingüísticas y antropológicas europeas contemporáneas, una particular apropiación -un uso- que los participantes en el debate hacen por ejemplo de las teorías de Meillet, Rivet o Saussure. A partir del análisis de estas intervenciones, la autora discute qué implicancias tiene la inscripción de la lingüística indígena en el ámbito de la americanística y su relación con el desarrollo de la lingüística como ciencia, y propone una interpretación acerca de las razones que permiten explicar esta particular distribución disciplinar.

A continuación, el artículo de Esteban Lidgett examina una serie de textos publicados entre 1904 y 1913 por Ricardo Monner Sans en dos revistas del período, la Revista de Historia, Derecho y Letras y la Revista de la Universidad de Buenos Aires. Así como el trabajo de Narvaja de Arnoux abordaba los manuales de retórica y buscaba reconstruir en relación con ellos un conjunto de intervenciones vinculadas a la lengua y sus usos, la contribución de Lidgett se detiene también en los debates educativos y examina el modo en que en la prensa periódica se discute respecto de la enseñanza de la lengua, y en particular acerca del lugar de la gramática en ese proyecto pedagógico. Para ello, el autor reconstruye en primer lugar las reformas educativas que como política lingüística estatal se diseñan entre fines del XIX y comienzos del XX, y observa que en su alternancia parecen responder a dos modelos, ambos concebidos sin embargo como respuesta al riesgo que, se presume, supone la inmigración como potencial disruptivo: por una parte, un modelo basado en una tradición gramatical escolar de carácter prescriptivo que se plantea como objetivo el resguardo de la lengua nacional ante las amenazas de "corrupción lingüística"; por otra parte, otro modelo que, intentando dar respuesta al conflicto pedagógico que suponía la enseñanza de la lengua, busca en la retórica y en la literatura una renovación de los contenidos y de los objetivos de la enseñanza. A través del estudio de esos textos que Monner Sans publica en la prensa periódica, Lidgett establece que Monner Sans defiende un modelo de enseñanza focalizado en los contenidos gramaticales y con una marcada orientación a privilegiar la función correctiva y ejemplificadora de esta disciplina. Su contribución constituye así un aporte al estudio global de las ideologías lingüísticas, que permite observar la conformación de un modelo de enseñanza de la lengua que pretende sintetizar la tradición gramatical escolar con un objetivo pedagógico concreto: la estigmatización de los usos lingüísticos que se apartan de la norma culta, en una clara vinculación con el discurso nacionalista prohispánico que emerge en la Argentina durante el período analizado. 
Diego Bentivegna explora, con las herramientas que proveen la glotopolítica, el análisis del discurso y los estudios literarios, en primera instancia un corpus preciso de una firma central en el campo de la literatura, la cultura y la política argentinas de comienzos del siglo XX. "Leopoldo Lugones: etimología y poder. Antecedencias y precedencias en La Nación (1923-1925)" se detiene así en el análisis de una serie de artículos publicados por el poeta de Lunario sentimental en las páginas del matutino porteño, situando estos textos en el complejo escenario de lectura que ofrecen la obra del propio Lugones, el periódico y la época. Como observa el autor, las notas etimológicas lugonianas son contemporáneas de la profundización del perfil autoritario y nacionalista en su discurso (paradigmáticamente expresado en el célebre discurso de Ayacucho), y en esa tónica sugiere observar en ellas un gesto glotopolítico fuerte que apunta a fortalecer el vínculo entre lengua y nación a partir del principio de la homogeneidad y el establecimiento de filiaciones prestigiosas que vinculen la propia idiosincrasia lingüística con un linaje prestigioso. Así, el léxico americano podría adquirir su legitimidad al filiarse con tradiciones letradas ancestrales y prestigiosas, y al mismo tiempo inmunizarse con respecto a las posibles contaminaciones o parentescos con los elementos provenientes de las culturas indígenas latinoamericanas. Leído al mismo tiempo en el contexto de la instalación del Instituto de Filología y de las diversas intervenciones acerca de la identidad lingüística americana -sobre todo las de la "serie euríndica" de Ricardo Rojas-, así como en el de la consolidación del fascismo europeo y sus sucedáneos transatlánticos, el esfuerzo filológico de Lugones cobra una significación más fuerte y compleja que la de la mera intervención pretendidamente erudita del escritor prestigioso. De este modo, el corpus inicial de las notas lugonianas se expande en un análisis que comprende aquellos textos que entran en un complejo diálogo polémico con ellas por entonces, firmados entre otros por Américo Castro, Menéndez Pidal, Paul Groussac, Ricardo Rojas y Rudolf Lenz, entre otros. El trabajo de Bentivegna ofrece así un examen riguroso de una intrincada trama de intervenciones glotopolíticas que tienen su escenario privilegiado en esos años en la prensa, particularmente en el diario de los Mitre.

El trabajo de Toscano y García estudia la encuesta que, en el año 1927, realiza el diario porteño Crítica con el objetivo de determinar la eventual existencia de un idioma argentino. Utilizando un mecanismo, el de la encuesta pública, del que había dispuesto y dispondría en numerosas oportunidades, la encuesta de Crítica es para Toscano y García al mismo tiempo que el relevamiento de un conjunto de saberes y creencias en relación con el futuro de la lengua una imagen del proceso que, contemporáneamente, se produce de conformación de un campo científico para los estudios lingüísticos. Así, el autor establece que la creación, en 1922, del Instituto de Filología de la Universidad de Buenos Aires supone un proceso de profesionalización de la reflexión lingüística, una de cuyas consecuencias es la progresiva funcionalización que se observa en el campo entre un saber experto y uno que no lo es. Toscano y García muestra que la encuesta se desarrolla en dos etapas: en la primera dominan un conjunto de intervenciones unificadas por la común ubicación periférica respecto del centro del campo científico; en la segunda aparece la voz experta de los filólogos españoles a cargo del Instituto de Filología. De este modo, la propia organización de la encuesta parece testimoniar ese proceso de reorganización y distinción de los campos que se observa durante la década del veinte. Al mismo tiempo, Toscano y García sostiene que esa distinción entre saber experto y no experto no es un buen predictor del espesor teórico de las intervenciones en el debate, ya que según demuestra algunos de los planteos que la lingüística profesional desarrollará durante la década del treinta se encuentran presentados en el primer tramo de la encuesta; las diferencias más significativas, sostiene, deben buscarse en el plano de las formas argumentativas por las que ambos grupos optan, pero fundamentalmente por la representación del problema de la lengua como un problema de carácter relativamente autónomo o de carácter estético.

El trabajo de Laura Sesnich, "Nivelar la lengua, consolidar un mercado: Amado Alonso y la 'época de oro' de la edición en Argentina” aborda en el análisis detenido de un corpus preciso y claramente delimitado -los artículos periodísticos publicados por Amado Alonso en La Nación en agosto de 1940- una problemática crucial para pensar la relación entre lengua y cultura impresa: el lugar de los proyectos de regulación de las prácticas lingüísticas en la época dorada del mercado editorial argentino. Directamente relacionado con 
la crisis general de la producción española en la guerra civil y su posguerra, el fenómeno de la formidable expansión de la industria editorial argentina que tiene lugar a partir de los últimos 30 cuenta entre sus actores decisivos con muchos españoles, exiliados o desplazados de diversos modos. Uno de ellos es Amado Alonso, director del Instituto de Filología desde 1927 y miembro fundador de la popular editorial Losada, quien en estos artículos desarrolla una completa propuesta de regulación lingüística para el mercado editorial en lengua española, que articula lengua, cultura, economía y política en una renovada versión del antiguo mandato de la unidad panhispánica.

Finalmente, en "Centro/periferia: la cuestión de la soberanía lingüística en La Carreta y en La prensa cegetista (Argentina, 1940-1941; 1952-1953)”, Mara Glozman propone un acceso a los discursos y debates sobre la lengua a partir de un principio de organización de sus materiales -de un modo de hacer archivoque atiende a aspectos particulares de su funcionamiento y del modo de articularse en la coyuntura. En este caso en particular, serán los ejes proporcionados por los pares de opuestos políticos soberania/emancipación y dependencia/dominación los que proporcionen un principio de construcción crítica para la lectura de un corpus de materiales de prensa, si bien inicialmente heterogéneos, decididamente compatibles en el modo de articularse en ellos lengua y política. En ambos casos, además, a pesar de las distancias evidentes, se observa la coincidencia en el carácter cultural de las publicaciones y su condición marginal o periférica con respecto a los medios investidos de una mayor legitimidad en el campo de la cultura. Las dos series textuales de La Carreta y La Prensa en su período a cargo de la CGT se inscriben en sendos momentos cruciales de los debates sobre la lengua en Argentina, a comienzos de los 40 y a comienzos de los 50, respectivamente, momentos en los que ambas publicaciones vuelven a traer a escena la vindicación de la soberanía lingüística en términos de gestión autónoma y no dependiente de los organismos españoles de la lengua legítima.

De esta manera, el monográfico propone un recorrido amplio por dos siglos de historia de la reflexión y el debate sobre la lengua en la prensa escrita, mayormente inscripto en Argentina, pero sin dejar de contemplar una dinámica transatlántica y continental. La prensa, por otra parte, y más ampliamente esa cultura impresa que hará posible la institución moderna de la literatura, no aparece como mero vehículo o soporte de la intervención letrada sobre el lenguaje, sino, como se ha visto, como nuevo medio en que la misma habita, se desarrolla, define y -sobre todo- se convierte en objeto de disputa.

\section{Bibliografía}

Acree, W. (2013). La lectura cotidiana. Cultura impresa e identidad colectiva en el Río de la Plata, 1780-1910. Buenos Aires: Prometeo.

Adamowicz-Hariasz, M. (1999). From Opinion to Information: The Roman-Feuilleton and the Transformation of the Nineteenth-Century French Press. En D. De la Motte y J. Przyblyski (eds.), Making the News. Modernity \& the Mass Press in Nineteenth-Century France. Amherst: University of Massachusetts Press, pp. 160-184.

Anderson, B. (1993) [1983]. Imagined Communities. Reflections on the Origin and Spread of Nationalism. Londres: Verso.

Arnoux, E. Narvaja de (2000). La glotopolítica: transformaciones de un campo disciplinar. En Lenguajes: teorias y prácticas. Buenos Aires: Gobierno de la Ciudad de Buenos Aires, Secretaría de Educación, pp. 95-109.

Arnoux, E. Narvaja de (2016). La perspectiva glotopolítica en el estudio de los instrumentos lingüísticos: aspectos teóricos y metodológicos. Matraga, 38, 18-42.

Arnoux, E. y del Valle, J. (2010). Las representaciones ideológicas del lenguaje. Discurso glotopolítico y panhispanismo. Spanish in Context, 7, 1, 1-24.

Auroux, S. (1994). La révolution technologique de la grammatisation. Lieja: Mardaga.

Auroux, S. (2009). Instrumentos lingüísticos y políticas lingüísticas: la construcción del francés. Revista Argentina de Historiografia Lingüistica I, 2, 137-149. 
Bierbach, M. (2000). Spanisch - eine plurizentrische Sprache? Zum Problem von norma culta und Varietät in der hispanophonen Welt. Vox Romanica 59, 143-170.

Blommaert, J. (2005). Discourse. A Critical Introduction. Nueva York y Londres: Cambridge University Press.

Blommaert, J. y Verschueren, J. (1998). The Role of Language in European Nationalist Ideologies. En B. Schieffelin, K. Woolard y P. Kroskrity (eds.). Language Ideologies. Practice and Theory. Nueva York y Oxford: Oxford University Press, pp. 189-210.

Blumenthal, P. (2003). Der Begriff der esternen und internen Sprachgeschichte in der Romanistik. En G. Ernst, M-D Gleßgen, C. Schmitt y W. Schweickard (eds.). Romanische Sprachgeschichte. Ein internationales Handbuch zur Geschichte der romanischen Sprachen. Berlín y Nueva York: de Gruyter.

Botrel, J-F. (1998). Teoría y práctica de la lectura en el siglo XIX: el arte de leer. Bulletin Hispanique, 100, 2, 577-590.

Bourdieu, P. (1982). Ce que parler veut dire. L'économie des échanges linguistiques. París: Fayard.

Bourdieu, P. (1992). Les Règles de l'art. Genèse et structure du champ littéraire. París: Seuil.

Burke, P. (2004). Languages and Communities in early Modern Europe. Cambridge: Cambridge University Press.

Clyne, M. (1992). Pluricentric Languages-Introduction. En M. Clyne (ed.). Pluricentric Languages. Differing Norms in Different Nations. Berlín y Nueva York: Mouton/de Gruyter, pp. 1-9.

Coseriu, E. (1978). Sincronia, diacronia e historia. El problema del cambio lingüistico. Madrid: Gredos.

Crowley, T. (1996). Language in History. Londres y Nueva York: Routledge.

Del Valle, J. (ed.) (2013). A political history of Spanish. Cambridge: Cambridge University Press.

Del Valle, J. (ed.) (2015). Historia politica del español. La creación de una lengua. Madrid: Aluvión.

Del Valle, J. y Gabriel-Stheeman, L. (eds.) (2002). The Battle over Spanish between 1800 and 2000. Language ideologies and Hispanic intellectuals. Londres y Nueva York: Routledge.

Eisenstein, E. (2005) [1983]. The Printing Revolution in Early Modern Europe. Cambridge: Cambridge University Press.

Ennis, J. A. (2008). Decir la lengua. Debates ideológico-lingüisticos en la Argentina desde 1837. Frankfurt am Main, Berlin, Bern, Bruxelles, New York, Oxford, Wien: Peter Lang.

Ennis, J. (2014). El uso, la propiedad y el valor en el debate por la lengua americana. Anclajes, 18, 19-34.

Ennis, J. (2017). Soberanía lingüística. Una discusión glotopolítica. En R. Bein, J. E. Bonnin, M. Di Stefano, D. Lauria, C. Pereira (eds.). Homenaje a Elvira Arnoux. Estudios de análisis del discurso, glotopolitica y pedagogía de la lectura y la escritura. Buenos Aires: FFyL-UBA, pp. 303-320.

Ennis, J. y Pfänder, S. (en prensa). El español de América entre oralidad y escritura, cultura letrada y cultura popular. En E. Ekkrammer (ed.). Manual del español de América. Berlín y Nueva York: de Gruyter.

Febvre, L. y Martin, H-J (1958). L'apparition du livre. París: Albin Michel.

Fontanella de Weinberg, M. B. (1992). El español de América. Madrid: Mapfre.

Gal, S. (1989). Language and Political Economy. Annual Review of Anthropology, 18, 345-367.

Garatea, C. (2006). Pluralidad de normas en el español de América. Revista Internacional de Lingüistica Iberoamericana, 4, 141-158.

Garatea, C. (2008). Unidad y diversidad en el español de América. En D. da Hora y R. Marques (eds.). Politica linguistica na America Latina. João Pessoa: Idéia.

García, O., Flores, N. y Spotti, M. (2017). Introduction: Language and society: a critical, poststructuralist perspective. En O. García, N. Flores y M. Spotti (eds.). The Oxford Handbook of Language and Society. Nueva York: Oxford University Press, pp. 1-16.

Greußlich, S. (2015). El pluricentrismo de la cultura lingüística hispánica: política lingüística, los estándares regionales y la cuestión de su codificación. Lexis, XXXIX, 1, 57-99.

Guitarte, G. (1983). Siete estudios sobre el español de América. México: UNAM.

Habermas, J. (1962). Strukturwandel der Öffentlichkeit. Neuweid: Luchterhand. 
Lebsanft, F. (1998). Spanische Sprachkultur - monozentrisch oder plurizentrisch?. En A. Greule y F. Lebsanft (eds.). Europäische Sprachkultur und Sprachpflege. Tubinga: Narr, pp. 255-276.

Heller, M. y McElhinny, B. (2017). Language, Capitalism, Colonialism. Towards Critical History. Toronto: Toronto University Press.

Kroskrity, P. (2006). "Language ideologies". En A. Duranti (ed.). A Companion to Linguistic Anthropology. Londres \& New York: Blackwell, pp.496-517.

Lebsanft, F. (2004). Plurizentrische Sprachkultur in der spanischsprachigen Welt. En A. Gil, D. Osthus y C. PolzinHaumann (eds.). Romanische Sprachwissenschaft. Zeugnisse fu\#r Vielfalt und Profil eines Fachs. Festschrift fu\#r Christian Schmitt zum 60. Geburtstag. Vol. 1. Fráncfort: Peter Lang, pp. 205-220.

Lebsanft, F. (2007). Norma pluricéntrica del español y Academias de la Lengua. En C. F. Laferl y B. Pöll (eds.). Amerika und die Norm. Literatursprache als Modell? Tubinga: Niemeyer, pp. 227-246

Lebsanft, F. et al. (2012). El español, ¿desde las variedades a la lengua pluricéntrica?. Madrid: Iberoamericana; Frankfurt am Main: Vervuert

Lotman, Y. M. (1988). Estructura del texto artístico. Madrid: Istrea.

Milroy, J. (2001). Language ideologies and the consequences of standardization. Journal of Sociolinguistics, 5, 4, 530-555.

Milroy, J. y Milroy, L. (1985). Authority in Language. Investigating Standard English. Londres y Nueva York: Routledge.

Smakman, D. (2012). The definition of the standard language: a survey in seven countries. International Journal of the Sociology of Language, 218, 25-58.

Viala, A. (1985). Naissance de l'écrivain: Sociologie de la littérature à l'âge classique. París: Minuit.

Williams, R. (1977). Marxism and Literature. Oxford: Oxford University Press.

\section{Notas}

1 Con respecto a la relación entre el desarrollo de las lenguas nacionales estándar modernas y el régimen taylorista de producción, v. Heller y McElhinny (2017:113-114).

2 Con respecto al español como lengua pluricéntrica puede verse Guitarte 1983, Fontanella de Weinberg 1992, Clyne 1992, Lebsanft 1998, 2004, 2007, Lebsanft et al.2012, Bierbach 2000, Garatea 2006, 2008, Ennis 2008, Greußlich 2015, entre otros. Acerca de la relación entre el pluricentrismo del español de América, la cultura impresa y la estandarización, se viene trabajando también en el marco de este proyecto, v. Ennis en prensa, Ennis y Pfänder en prensa.

3 Ver, entre otros, Arnoux y del Valle 2010, Arnoux 2000, 2016, Del Valle 2007, 2013, 2016.

4 Optamos aquí por el término "audiencia", que para esa etapa de formación aparece como más pertinente, porque permite pensar en sujetos lectores y también, en los lectores-oyentes (Botrel 1998), es decir en aquellos que, dominantemente en el XIX, no tienen las competencias específicas ni el acceso a los materiales de las elites letradas pero no dejan por ello de ser "lectores". Sobre la relación entre lengua y soberanía (v. Ennis 2017).

5 García, Flores y Spotti (2017:13) establecen recientemente un vínculo interesante entre la perspectiva de las ideologías lingǘsticas y la pregunta postestructuralista por la governance: "dominant language ideologies have been complicit in the formation of governable subject positions both historically and in our current neoliberal context".

6 Teniendo en cuenta, claro está, salvedades como las interpuestas por Eisenstein, el desarrollo tecnológico, económico y político que hace posible la expansión de la cultura impresa resultará decisivo en la formación del estado-nación moderno -como puede leerse ya en la explicación del desarrollo del print capitalism que proporciona Anderson (1983), en cuya lógica la lengua (de la prensa, de la novela, de los manuales escolares), que será a su vez la de la educación y la administración, juega un rol determinante. (Eisenstein 2005: 5-6: "it is one thing to show that standardization was a consequence of printing. It is another to decide how laws, languages, or mental constructs were affected by more uniform texts").

7 Con instrumentos lingüisticos nos referimos aquí al estudio de manuales, gramáticas escolares, diccionarios (v. Auroux 1994, 2009, Arnoux 2016). 